\title{
Periodic Visuotactile Stimulation Slowly Enhances the Rubber Hand Illusion in Individuals with High Autistic Traits
}

\author{
Masakazu Ide ${ }^{1,2}$ and Makoto Wada ${ }^{1 *}$ \\ ${ }^{1}$ Developmental Disorders Section, Department of Rehabilitation for Brain Functions, Research Institute of National \\ Rehabilitation Center for Persons with Disabilities, Saitama, Japan, ${ }^{2}$ Japan Society for the Promotion of Science, Tokyo, \\ Japan
}

OPEN ACCESS

Edited by:

Emiliano Macaluso,

University Claude Bernard Lyon 1,

France

Reviewed by:

Massimiliano Zampini,

University of Trento, Italy

Maria Laura Filippetti,

Royal Holloway University, UK

*Correspondence:

Makoto Wada

wada-makoto@rehab.go.jp

Received: 12 February 2016

Accepted: 26 May 2016

Published: 09 June 2016

Citation:

Ide M and Wada M (2016) Periodic

Visuotactile Stimulation Slowly

Enhances the Rubber Hand Illusion in

Individuals with High Autistic Traits.

Front. Integr. Neurosci. 10:21

doi: 10.3389/fnint.2016.00021
In a rubber hand illusion $(\mathrm{RH})$ task, synchronous brush stroking of a rubber hand and a participant's hidden hand induces body ownership of the rubber hand. The effects of spatial distances and temporal lags on the $\mathrm{RHI}$ have been extensively examined; however, the effect of periodicity of the stimuli on illusory body ownership has not been examined. Meanwhile, the occurrence of $\mathrm{RHI}$ tends to be weak in individuals with autism-spectrum disorders (ASD) and high autistic traits. Preference for stimulus having regularity of tempo is generally observed in individuals with ASD, and thus, periodic stimulation might be more effective to elicit the body ownership illusion in individuals with high autistic traits. Hence, we investigated whether stimulus periodicity influenced $\mathrm{RHI}$ as well as its association with participant's autistic traits. Brush strokes were applied to a participant's own hand and the rubber hand periodically (2 s) or non-periodically (1-3 s), either synchronously or asynchronously. Two blocks were performed in each condition. We found that periodic stimulation enhanced the spatial updating of tactile sensation induced by $\mathrm{RHI}$ in the subsequent block in participants with high autistic traits, whereas both periodic and non-periodic stimulation strongly elicited $\mathrm{RHI}$ in blocks 1 and 2. These results indicate that the periodicity of stimulation has different effects based on an individual's autistic traits. Since individuals with ASD are known to sustain their focus on interoceptive sensations (heartbeats), a periodic stimulation that is potentially correlated with heartbeats might be effective to enhance the visuotactile integration during $\mathrm{RHI}$ in individuals with high autistic traits.

Keywords: stimulus periodicity, rubber hand illusion, autistic traits, interoceptive sensation, visuotactile integration, body representation

\section{INTRODUCTION}

In a rubber hand illusion (RHI), a participant's hidden hand and a rubber hand are synchronously stroked by paintbrushes, and an individual gradually feels as if the rubber hand is his or her own hand (Botvinick and Cohen, 1998). Concurrently, participants feel that their own hands are more toward the rubber hand (proprioceptive drift). Spatiotemporal congruency between visual and tactile (visuotactile) stimuli is considered a crucial factor for RHI. Consistent with this notion, 
the distance between the participant's hand and the rubber hand should be within a certain range on both horizontal (Lloyd, 2007) and vertical axes (Kalckert and Ehrsson, 2014) to induce RHI. In addition, anatomical plausibility of the hand also affects RHI because the illusion occurs only when the joint angles are within ranges in which participants can mimic the posture (Tsakiris and Haggard, 2005; Ide, 2013). Regarding temporal limitations of the illusion, visuotactile stimuli must be presented within a given amount of temporal lag ( 300 ms) (Shimada et al., 2009, 2014). Thus, congruent spatiotemporal stimuli can selectively induce strong RHI.

In these previous studies, the effect of the spatial proximity and synchronicity of the stimuli have been examined, but periodicity of the stimulus has been never considered. In previous experiments, manual stimulation was delivered in constant/periodical intervals $\sim 0.5-1 \mathrm{~Hz}$ (Ehrsson et al., 2005; Shimada et al., 2009; Suzuki et al., 2013). Since interoceptive sensation (e.g., heartbeats) also has periodicity $(\sim 1 \mathrm{~Hz})$, there is a commonality between the periodicity of stimulation to induce RHI and that of heartbeats. Recent studies have suggested that visual feedback of heartbeats on the rubber hand (change in color; Suzuki et al., 2013) or a full body image (flashes; Aspell et al., 2013) induces illusory body ownership. To our knowledge, no study is available that directly compared the effect of stimulus periodicity (periodic or non-periodic) on illusory body ownership. Thus, it is intriguing to examine the effect of periodicity of synchronous stimuli on the illusory sensation during the RHI task, since such a periodic feature of stimulation might play an important role in inducing the ownership of the rubber hand.

Meanwhile, individuals with autism-spectrum disorders (ASD) have several symptoms such as severe and sustained impairment in social interaction, deviance in communication, and restricted interests or repetitive behavior patterns. It is known that they process information from different sensory modalities in atypical styles compared with neurotypical persons (Kanner, 1943; Marco et al., 2011). Recent studies have started to reveal diverse characteristics regarding their sensory processing, especially for multisensory integration and interaction with the external environment. For example, the temporal binding window of audiovisual stimuli is larger in individuals with ASD compared with neurotypical persons (Foss-Feig et al., 2010; Kwakye et al., 2011). Moreover, diversities of illusory body ownership during RHI in individuals with ASD indicate abnormalities in visuotactile integration. In a reach-to-grasp movement subsequent to the induction of RHI, the normalized integrated jerk of reach-to-grasp movements significantly increased in neurotypical persons, whereas execution of the movement was not affected by the illusion in individuals with ASD (Palmer et al., 2015a). Delayed occurrence of the proprioceptive drift during RHI was also reported, such that the drift was not observed until a subsequent block. In contrast, the proprioceptive drift was obvious in the first block in neurotypical persons (Cascio et al., 2012). Similar features were also seen in individuals with high autistic traits without a diagnosis of ASD. They tended to exhibit small proprioceptive drifts during RHI (Paton et al., 2012; Palmer et al., 2013). Our recent study has suggested that subjective feeling of ownership of the rubber hand can be also predicted by individual's autistic traits, especially the degree of social communication ability (Ide and Wada, 2016). Thus, individuals with high autistic traits seem to have common properties to those of individuals with ASD regarding the features of body ownership.

Autistic traits form a wide spectrum, and several such traits can be seen even in nonclinical persons (Palmer et al., 2015b). Thus, in line with the previous study with individuals with ASD (Cascio et al., 2012), we predicted that delayed occurrence of RHI would be observable in individuals with high autistic traits. In this study, we investigated the effect of stimulus periodicity on the RHI. Previously, in individuals with ASD, preference for stimulus with regularity of tempo was observed (Hutt and Hutt, 1968; Frankel et al., 1978; Garretson et al., 1990). We assumed that this preference for regularity of an external stimulus might reflect their enhanced ability to sustain their own interoceptive sensation, because it has been reported that external stimulus which is synchronously delivered with participants' own heartbeats selectively attracts their attention (Kroeze and van den Hout, 2000; Pineles and Mineka, 2005). Thus, we hypothesized that periodic stimulation might be more effective to elicit illusory body ownership in individuals with high autistic traits than with low autistic traits. In the present study, we investigated whether periodic stimulation dominantly enhanced the illusory body ownership in nonclinical individuals with high autistic traits using PC-controlled machinery stimulators that can easily control the timing of the stimulus.

\section{MATERIALS AND METHODS \\ Participants}

Twenty-two participants (18 females, 4 males; $23 \pm 2.9$ years old) participated in this study after we excluded two participants who continuously failed to perform the proprioceptive drift task. All participants had normal or corrected-to-normal (e.g., glasses, contacts) vision and no history of neurological diseases. We scored their handedness according to the Edinburgh Inventory (Oldfield, 1971). In total, 19 participants were right-handed $(+50<$ L.Q. $<+100,84.7 \pm 15.8)$, two participants were ambidextrous (L.Q. $=10,0$, respectively), and one participant was left-handed (L.Q. $=-30$ ). Additionally, we assessed the participants' autistic traits using the Japanese version of the Autism Spectrum Quotient (AQ) score (Wakabayashi et al., 2004), which was translated from the English version (BaronCohen et al., 2001). The mean AQ score of the participants was $17.3 \pm 3.63$ (mean $\pm S D$ ). The ethics committee of the National Rehabilitation Center for Persons with Disabilities approved the study; informed consent was obtained from all participants before the experiments.

\section{Stimulus}

A rubber hand (Otto Bock, Duderstadt, Germany) was placed on the desk in front of the participant (Figure 1). For brush stroking, we used a customized device with two brushes that 


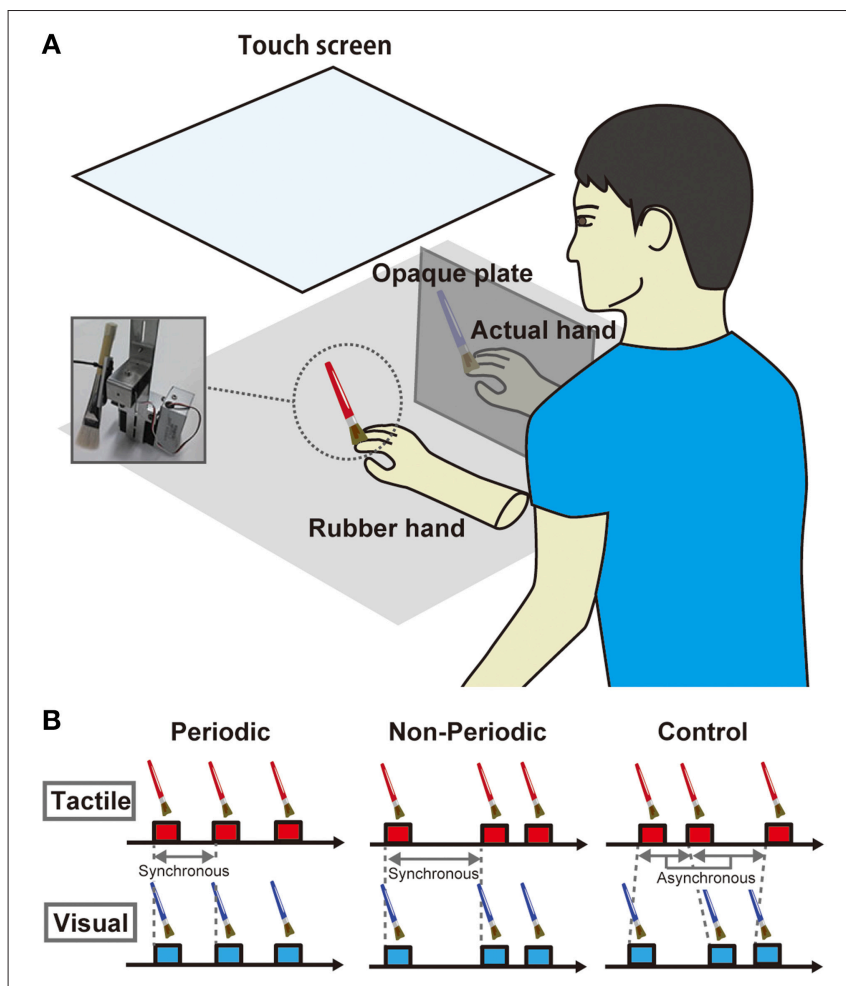

FIGURE 1 | (A) Task setting. A rubber hand was placed on the desk in front of the participants. Brush stroking was delivered to the rubber hand and the participant's own hand using PC-controlled machinery stimulators. A touch screen was placed above the rubber hand and the participant's hand while the participant could see the rubber hand. (B) Task conditions. There were three conditions in terms of the temporal parameters of stimulus periodicity. In the periodic condition, the intervals of synchronous brush stroking to the rubber and actual hands were constant at $2 \mathrm{~s}$. In the non-periodic condition, the intervals were randomly assigned and ranged from 1 to $3 \mathrm{~s}$. In the asynchronous condition, the intervals were individually and randomly assigned to the rubber hand and actual hand and ranged from 1 to $3 \mathrm{~s}$.

were attached on DC motors (MS025, Solidray Co Ltd, Japan); the timings of the stimuli were arbitrarily assigned by a personal computer (VAIO, SONY, Japan) with Matlab software (MathWorks, Natick, MA, USA). Strokes from the reciprocating brushes were synchronously delivered to the rubber hand and the participant's own hand at a velocity of maximum $545.5^{\circ} / \mathrm{s}$ $(80 \mathrm{~cm} / \mathrm{s})$. A touch screen (KTMT-1921 Add-On Touch Screen for 20-21" LCD \& CRT, KEYTEC, Inc., Garland, TX, USA) was placed above the rubber hand and the participant's hand while the participant could see the rubber hand. The participants wore earplugs and noise-canceling headphones to block sounds generated by the motor.

\section{Procedure}

The rubber hand was placed in front of the participant while her/his right hand was placed on the right side of the rubber hand (13 cm from the rubber hand); the right hand was hidden from view by an opaque plate. We asked the participant to fixate on an index finger of the rubber hand where the brush strokes were delivered. We adopted three conditions regarding the temporal parameters of the visuotactile stimulation (Figure 1B). In the periodic condition, intervals of synchronous brush stroking on the rubber and actual hands were constant at $2 \mathrm{~s}$. In the nonperiodic condition, the intervals were randomly assigned within 1 to $3 \mathrm{~s}$, while the total numbers of stimuli were the same as that in the periodic condition, and the mean interval in the nonperiodic condition was $\sim 2 \mathrm{~s}$. In the asynchronous condition, the intervals were individually and randomly assigned to the rubber hand and the actual hand within a range of 1 to $3 \mathrm{~s}$. There were six stimulation blocks in total, so we performed each condition twice. One block requires $3 \mathrm{~min}$, and the entire experiment, including rest periods between blocks, was performed in $30 \mathrm{~min}$.

We asked the participants to perform two tasks to measure the degree of RHI. First, the participants answered the degree of their subjective feeling of RHI in questionnaires that were translated into Japanese Likert 7-point scale from +3 (agree strongly) to -3 (disagree strongly; Table 1). In the questionnaires, we assumed that only three of nine items relate to the subjective feeling of RHI (Botvinick and Cohen, 1998). Second, the participants were required to use their left hand to point at the place on the touch screen where they felt their right index finger. We calculated the amount of proprioceptive drift by subtracting the horizontal position indicated prior to the block from the position indicated after the block.

Table 2 indicates mean scores of subjective feeling of RHI that was assessed by the three questionnaires and proprioceptive drifts in each block of each stimulus condition. We defined change in subjective feeling of RHI (Sync-Async) by subtracting individual scores in items 1, 2, and 3 on the questionnaires (see Table 1) in the asynchronous condition from those scores in synchronous conditions (periodical and non-periodical conditions). We averaged the scores from two blocks of each condition. Similarly, we calculated change in the proprioceptive drift (Sync-Async) by subtracting the amount of proprioceptive drift in the asynchronous condition from the amount in the synchronous conditions.

\section{RESULTS}

In the behavioral test, most participants (21 out of the 22 participants) reported that they felt RHI during both periodic and non-periodic (but synchronous) stimulations (Table 2). Most participants felt stronger subjective feeling of RHI as indicated by at least one of the three items in synchronous conditions (periodical and non-periodical) compared with asynchronous condition.

\section{Effect of Periodic Stimulation on RHI in the Entire Group}

To examine the effects of stimulus periodicity on RHI, we conducted two-way repeated measures ANOVA with stimulus periodicities (periodical or non-periodical) and blocks (first or second) in the change in subjective feeling of RHI (item 1, 2, and 3 , respectively) of the entire group $(N=22)$. We did not identify a significant main effect for all items of the periodical conditions 
TABLE 1 | Questionnaires for subjective feeling of RHI (Botvinick and Cohen, 1998).

\begin{tabular}{|c|c|}
\hline Items & Text \\
\hline Item 1 & $\begin{array}{l}\text { It seemed as if I were feeling the touch of the paintbrush in the location where I saw the rubber hand touched. } \\
\text { ラバーハンドの位置で自分の手が触られている感覚が生じているように感じる。 }\end{array}$ \\
\hline Item 2 & $\begin{array}{l}\text { It seemed as though the touch I felt was caused by the paintbrush touching the rubber hand. } \\
\text { ラバーハンドを触っている筆が自分の手を触っているように感じる。 }\end{array}$ \\
\hline Item 3 & $\begin{array}{l}\text { I felt as if the rubber hand were my hand. } \\
\text { ラバーハンドがあたかも自分の手のように感じる。 }\end{array}$ \\
\hline Item 4 & $\begin{array}{l}\text { I felt as if my hand were drifting toward the rubber hand. } \\
\text { 自分の手がラバーハンド側に移動したように感じる。 }\end{array}$ \\
\hline Item 5 & $\begin{array}{l}\text { It seemed as if I might have more than one left hand. } \\
\text { 右手がもう一本存在しているかのように感じる。 }\end{array}$ \\
\hline Item 6 & $\begin{array}{l}\text { It seemed as if the touch I was feeling came from somewhere between my own hand and the rubber hand. } \\
\text { 自分の手とラバーハンドの間のどこかから触られているように感じる。 }\end{array}$ \\
\hline Item 7 & $\begin{array}{l}\text { I felt as if my hand were turning rubbery. } \\
\text { 自分の手がゴムでできているように感じる。 }\end{array}$ \\
\hline Item 8 & $\begin{array}{l}\text { It appeared as if the rubber hand were drifting toward my hand. } \\
\text { 右手がもう一本存在しているかのように感じる。 }\end{array}$ \\
\hline Item 9 & $\begin{array}{l}\text { The rubber hand began to resemble my own hand, in terms of shape, skin tone, freckles, or some other visual feature. } \\
\text { ラバーハンドの形や色などの特徵が自分に似ているように感じる。 }\end{array}$ \\
\hline
\end{tabular}

We assumed that only three of nine items in the questionnaire are related to the subjective feeling of RHI. The questionnaire was translated into Japanese.

TABLE 2 | Mean scores ( $($ SEM) for questionnaires relating the subjective feeling of RHI (item 1, 2, and 3) and proprioceptive drift.

\begin{tabular}{|c|c|c|c|c|c|c|c|c|}
\hline & \multicolumn{2}{|c|}{ Item 1} & \multicolumn{2}{|c|}{ Item 2} & \multicolumn{2}{|c|}{ Item 3} & \multicolumn{2}{|c|}{ Proprioceptive drift } \\
\hline & Block 1 & Block 2 & Block 1 & Block 2 & Block 1 & Block 2 & Block 1 & Block 2 \\
\hline Periodic & $1.68(0.36)$ & $1.59(0.39)$ & $1.86(0.25)$ & $2.05(0.26)$ & $1.86(0.27)$ & $1.81(0.18)$ & $1.81(0.87)$ & $3.02(0.79)$ \\
\hline Non-Periodic & $1.59(0.35)$ & $0.95(0.86)$ & $2.14(0.23)$ & $1.64(0.31)$ & $1.95(0.25)$ & $1.55(0.28)$ & $1.73(0.81)$ & $3.41(0.92)$ \\
\hline Asynchronous & $-0.77(0.47)$ & $-0.73(0.49)$ & $-0.82(0.39)$ & $-0.82(0.39)$ & $-0.55(0.45)$ & $-0.5(0.31)$ & $0.11(0.88)$ & $1.41(0.51)$ \\
\hline
\end{tabular}

$\left[F s_{(1,21)}=2.54,0.16\right.$, and $0.23 ; p s=0.13,0.69$, and 0.64 ; partial $\eta^{2}=0.11,0.01,0.01$; items 1,2 , and 3, respectively] and blocks $\left[F s_{(1,21)}=2.34,0.7\right.$, and $1.42 ; p s=0.14,0.41$, and 0.25 ; partial $\eta^{2}$ $=0.1,0.03,0.07$; items 1, 2, and 3, respectively] (Figure 2A) based on the defined criteria (Cohen, 1988; Ellis, 2010). We also did not identify any significant interactions among periodical conditions and blocks in all items $\left[F s_{(1,21)}=2.78,1.49\right.$, and 3.65; $p s=0.11$, 0.24 , and 0.69 ; partial $\eta^{2}=0.12,0.15,0.07$; items 1,2 , and 3 , respectively].

We also conducted two-way repeated measures ANOVA with stimulus periodicity types (periodical or non-periodical) and blocks (first or second) in change in the proprioceptive drift. We found that participants started to perceive their own right hand's position to be near the rubber hand in the second block compared with the first block $\left[F_{(1,21)}=6.41, p<\right.$ 0.05; partial $\eta^{2}=0.23$ ] (Figure 2B). However, we did not identify any significant effect of periodical conditions $\left[F_{(2,42)}=\right.$ 2.61, $p=0.09$; partial $\left.\eta^{2}=0.005\right]$ or an interaction between periodical conditions and blocks $\left[F_{(1,21)}=0.17, p=0.68\right.$; partial $\left.\eta^{2}=0.01\right]$.

\section{Influence of a Participant's Autistic Traits on the Temporal Transition of RHI}

We examined whether the temporal transition of subjective feeling of RHI and proprioceptive drift correlated with each participant's autistic traits. To estimate the temporal transition of $R H I$, we subtracted an individual's scores regarding the change in subjective feeling of RHI in the first block of each stimulus periodicity (periodic/non-periodic) from the scores in the second block (Block 2-1). We found that the AQ score positively correlated with the magnitude of the temporal transition of $\mathrm{RHI}$ in item 2 in periodic condition $(r=0.44, p<0.05)$ but not in non-periodic condition (Figure 3). No significant correlation was observed for items 1 and 3 in periodic and non-periodic conditions. Regarding the proprioceptive drift, we also subtracted the change in proprioceptive drift in the first block from that in the second block (temporal transition of proprioceptive drift [Block $2-1]$ ). We did not identify any correlation between the AQ score and the amount of temporal transition in proprioceptive drift for items 1,2 , and 3 ( $r s=-0.06,-0.2,0.22$, and $p s=0.78,0.36,0.31$, respectively). 

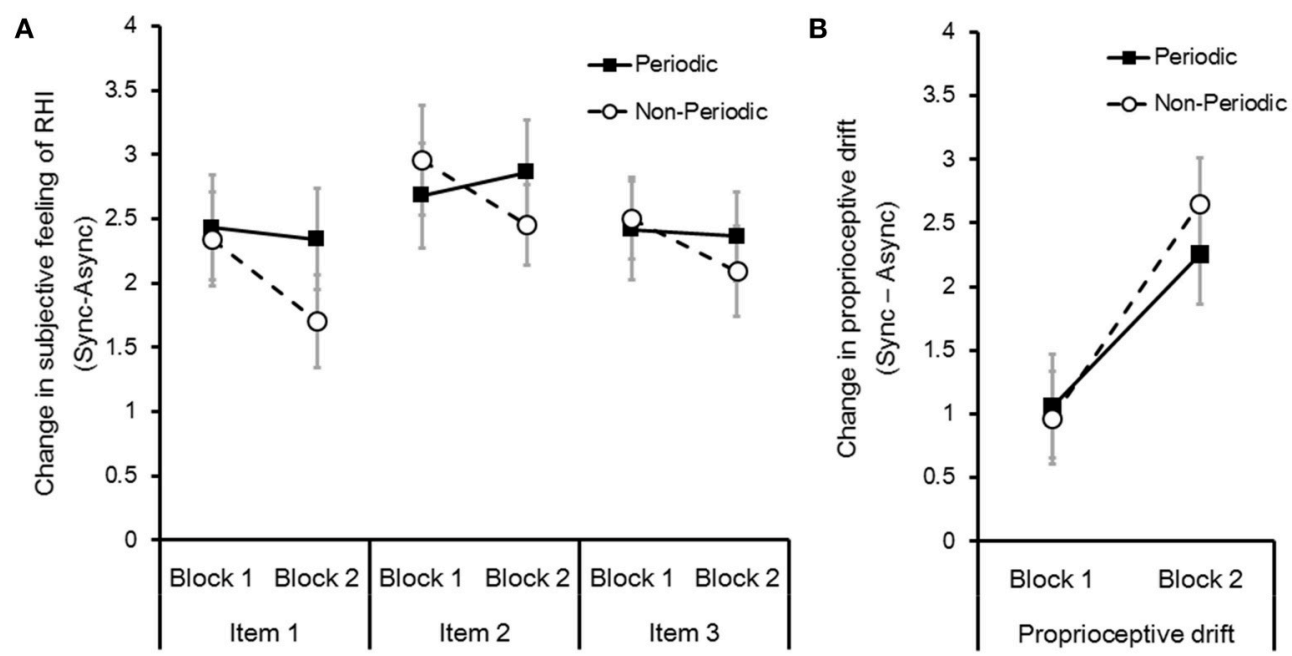

FIGURE 2 | (A) Effect of stimulus periodicity on the change in subjective feeling of RHI (Sync-Async). Change in subjective feeling of RHI were calculated for items 1 , 2, and 3 in the questionnaire (see Table $\mathbf{1}$ ) and blocks 1 and 2. Error bars denote the standard error of the mean $(N=22)$. (B) Effect of the stimulus periodicity on change in the proprioceptive drift (Sync-Async).
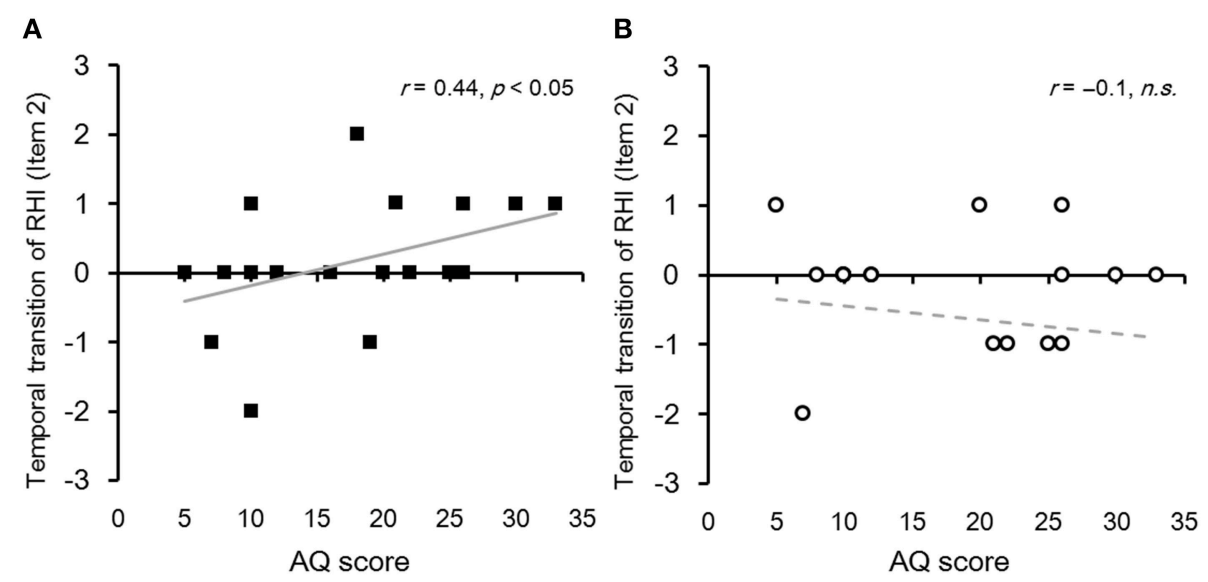

FIGURE 3 | (A) Relationship between the temporal transition of RHI and the participant's autistic traits in the periodic condition as assessed by item 2. Each black square indicates each participant's score regarding the temporal transition of change in subjective feelings of RHI. A sloped solid gray line indicates a regression linear function fitted with the least-squares method $(N=22)$. (B) Relationship between the temporal transition of RHI and the participant's autistic traits in the non-periodic condition assessed by item 2. Each open circle indicates each participant's score for temporal transition of change in subjective feelings of RHI. A sloped dotted gray line indicates a regression linear function fitted with the least-squares method $(N=22)$.

These results, from all participants $(N=22)$, indicated that the subjective feeling of spatial updating of tactile sensation (item 2) gradually increased in individuals with high autistic traits. Thus, we decided to further analyse item 2 to confirm whether the participant's autistic traits influenced the temporal transition of subjective feeling of RHI. To investigate the influence of autistic traits, we divided all participants $(N=22)$ into two groups based on the AQ scores (range: $5-33, M=17.82$, $S D=3.63$ ). Nine participants with low AQ scores were classified as the low AQ group (range: $5-12, M=10.73, S D=3.7$ ), and nine participants with high AQ scores were classified as the high AQ group (range: $21-33, M=22.1, S D=3.01$ ). Four participants were excluded from this analysis because they had average AQ scores (range: $16-20, M=17, S D=1.71$ ). A three-way one repeated measures ANOVA among groups (high AQ/low AQ), stimulus periodicities (periodical/non-periodical), and blocks (first/second) exhibited a significant interaction effect $\left[F_{(1,16)}\right.$ $=6.04, p<0.05]$ (Figure 4). Effect size of the interaction was small (partial $\eta^{2}=0.27$ ). A post-hoc test (Ryan, $p<0.05$ ) revealed that the subjective feeling of RHI in the periodical condition in the high AQ group increased in block two in contrast to the non-periodical condition (Cohen's $d=1.25$ ). However, in the low AQ group, stimulus periodicity did not have any effect on the subjective feeling of RHI in both blocks 1 and 


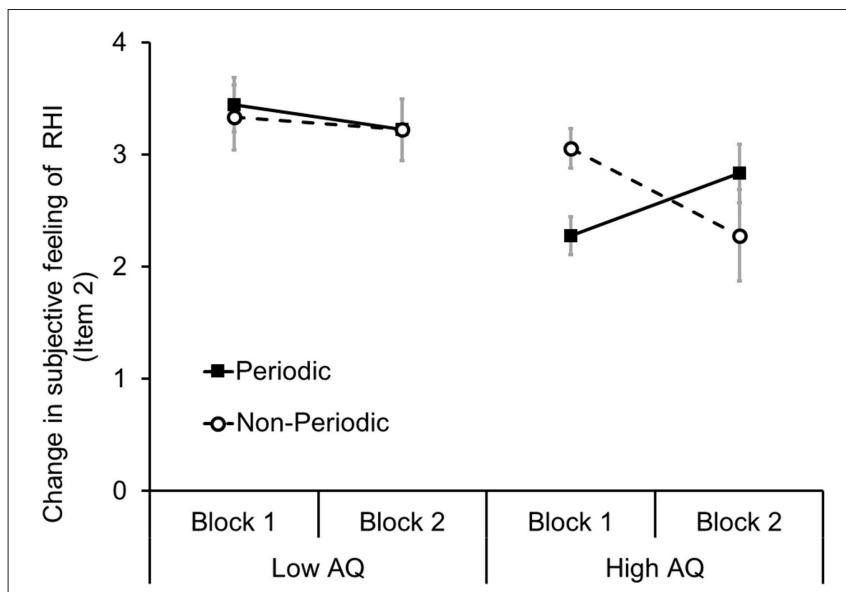

FIGURE 4 | Effect of stimulus periodicity on spatial updating of tactile sensation (item 2) of $\mathbf{R H I}$ in the low $A Q$ and high $A Q$ groups. A solid line with black squares indicates the mean of each participant's subjective feeling of $\mathrm{RHI}$ in the periodic condition, and a dotted line with white circle indicates the mean in the non-periodic condition. Error bars denote the standard error of the mean.

2. In addition, we did not identify such a tendency in items 1 and 3 .

\section{DISCUSSION}

In the present results, periodic visuotactile stimulation gradually promoted the spatial updating of tactile sensation in individuals with high autistic traits. In contrast, difficulty in feeling the illusory body ownership during RHI in individuals with ASD has been previously reported. Proprioceptive drift during RHI was not observed until a subsequent block in such participants (Cascio et al., 2012). Similar features were also seen in individuals with high autistic traits without a diagnosis of ASD. They tended to show small proprioceptive drift during RHI (Paton et al., 2012; Palmer et al., 2013). Current results showed that periodic stimulation could gradually enhance spatial updating of tactile sensation by RHI in individuals with high autistic traits.

Previous reports have demonstrated that visual feedback along with interoceptive information affects the induction of illusory body ownership (Aspell et al., 2013; Suzuki et al., 2013). It has also reported that the accuracy for interoceptive information (e.g., heartbeats) negatively correlates with the degree of illusory body ownership (Tsakiris et al., 2011). Individuals with ASD can sustain interoceptive awareness of their own heartbeats compared with neurotypical persons, and such an increased sustaining time is associated with the degree of RHI (Schauder et al., 2015). It is possible that the ability to focus on one's own internal bodily signals and external signals might exhibit a trade-off relationship. In this context, individuals with ASD (or high autistic traits) might also persist with their focus on an external object (e.g., the rubber hand), if the stimuli having similar periodicity to their own interoception (e.g., heartbeats) were provided. In the present study, periodic stimulation $(0.5 \mathrm{~Hz})$ was not presented in completely consistent way to participant's heartbeats, but it would be partly correlated with heartbeats' periodicity $(\sim 1 \mathrm{~Hz})$. We speculate that periodical stimulation could gradually facilitate spatial updating of tactile sensation in individuals with high autistic traits, though these participants are known to have difficulty in the occurrence of RHI (Paton et al., 2012; Palmer et al., 2013; Ide and Wada, 2016). To confirm this hypothesis, further experiments using brush stroking with timings of the participant's own heartbeats are needed. In the present study, in both periodic and non-periodic stimulation conditions, synchronous brush stroking elicited the subjective feeling of RHI compared with asynchronous brush stroking. However, with regard to item 2 in the questionnaire, we found that periodic stimulation enhanced the subjective feeling of RHI more in block 2 in individuals with high autistic traits. In item 2 ("It seemed as though the touch I felt was caused by the paintbrush touching the rubber hand."), it could be presumed that this sensation involved a degree of spatial updating of the tactile sensation from their actual hand to the rubber hand rather than illusory body ownership of the rubber hand. On the other hand, we did not observe such tendency in the spatial change of the feeling (item 1) and illusory ownership of the rubber hand (item 3).

In summary, the current results demonstrate that periodic brush stroking enhances the spatial updating induced by RHI in a delayed fashion in individuals with high autistic traits. These effects may be derived from their sustained awareness of interoceptive sensation (i.e., heartbeats). However, since the results are confined to the subjective feeling of RHI, especially about spatial updating of the rubber hand, this is not applicable to other aspects of illusory body ownership sensation and proprioceptive drift. Thus, further studies are needed to reveal the mechanism by which such strong awareness of interoceptive sensation in individuals with ASD could influence their cognition of external environments that have periodic or non-periodic features in daily life.

\section{AUTHOR CONTRIBUTIONS}

MI conducted the experiments; MW developed the apparatus; MW and MI designed the experiments and wrote the paper.

\section{ACKNOWLEDGMENTS}

We thank Prof. Satoshi Shibuya, Dr. Hanako Ikeda, Dr. Takeshi Atsumi for their helpful comments and Dr. Yasoichi Nakajima for his continuous encouragement. This study was supported by a Grant-in-Aid for Young Scientists (A) from JSPS (no. 26700012, 15K12615), MEXT (no. 15H01590) and a research grant from the HAYAO NAKAYAMA Foundation for Science and Technology and Culture to MW. 


\section{REFERENCES}

Aspell, J. E., Heydrich, L., Marillier, G., Lavanchy, T., Herbelin, B., and Blanke, O. (2013). Turning body and self inside out: visualized heartbeats alter bodily self-consciousness and tactile perception. Psychol. Sci. 24, 2445-2453. doi: 10.1177/0956797613498395

Baron-Cohen, S., Wheelwright, S., Skinner, R., Martin, J., and Clubley, E. (2001). The autism-spectrum quotient (AQ): evidence from Asperger syndrome/highfunctioning autism, males and females, scientists and mathematicians. J. Autism Dev. Disord. 31, 5-17. doi: 10.1023/A:1005653411471

Botvinick, M., and Cohen, J. (1998). Rubber hands 'feel' touch that eyes see. Nature 391, 756. doi: 10.1038/35784

Cascio, C. J., Foss-Feig, J. H., Burnette, C. P., Heacock, J. L., and Cosby, A. A. (2012). The rubber hand illusion in children with autism spectrum disorders: delayed influence of combined tactile and visual input on proprioception. Autism 16, 406-419. doi: 10.1177/1362361311430404

Cohen, J. (1988). Statistical Power Analysis for the Behavioral Sciences. Hillsdale: L. Erlbaum Associates.

Ehrsson, H. H., Holmes, N. P., and Passingham, R. E. (2005). Touching a rubber hand: feeling of body ownership is associated with activity in multisensory brain areas. J. Neurosci. 25, 10564-10573. doi: 10.1523/JNEUROSCI.0800-05.2005

Ellis, P. D. (2010). The Essential Guide to Effect Sizes: Statistical Power, MetaAnalysis, and the Interpretation of Research Results. New York, NY: Cambridge University Press.

Foss-Feig, J. H., Kwakye, L. D., Cascio, C. J., Burnette, C. P., Kadivar, H., Stone, W. L., et al. (2010). An extended multisensory temporal binding window in autism spectrum disorders. Exp. Brain Res. 203, 381-389. doi: 10.1007/s00221010-2240-4

Frankel, F., Freeman, B. J., Ritvo, E., and Pardo, R. (1978). The effect of environmental stimulation upon the stereotyped behavior of autistic children. J. Autism Child. Schizophr. 8, 389-394. doi: 10.1007/BF01538044

Garretson, H. B., Fein, D., and Waterhouse, L. (1990). Sustained attention in children with autism. J. Autism Dev. Disord. 20, 101-114. doi: 10.1007/BF02206860

Hutt, S. J., and Hutt, C. (1968). Stereotypy, arousal and autism. Hum. Dev. 11, 277-286. doi: 10.1159/000270612

Ide, M. (2013). The effect of "anatomical plausibility" of hand angle on the rubber-hand illusion. Perception 42, 103-111. doi: 10.1068/p7322

Ide, M., and Wada, M. (2016). Associations between salivary oxytocin concentration and subjective feeling during rubber hand illusion. J. Physiol. Sci. 66, S175.

Kalckert, A., and Ehrsson, H. H. (2014). The spatial distance rule in the moving and classical rubber hand illusions. Conscious. Cogn. 30, 118-132. doi: 10.1016/j.concog.2014.08.022

Kanner, L. (1943). Autistic disturbances of affective contact. Nervous Child 2, 217-250.

Kroeze, S., and van den Hout, M. A. (2000). Selective attention for cardiac information in panic patients. Behav. Res. Ther. 38, 63-72. doi: 10.1016/S00057967(99)00023-6

Kwakye, L. D., Foss-Feig, J. H., Cascio, C. J., Stone, W. L., and Wallace, M. T. (2011). Altered auditory and multisensory temporal processing in autism spectrum disorders. Front. Integr. Neurosci. 4:129. doi: $10.3389 /$ fnint.2010.00129

Lloyd, D. M. (2007). Spatial limits on referred touch to an alien limb may reflect boundaries of visuo-tactile peripersonal space surrounding the hand. Brain Cogn. 64, 104-109. doi: 10.1016/j.bandc.2006.09.013
Marco, E. J., Hinkley, L. B., Hill, S. S., and Nagarajan, S. S. (2011). Sensory processing in autism: a review of neurophysiologic findings. Pediatr. Res. 69, 48R-54R. doi: 10.1203/PDR.0b013e3182130c54

Oldfield, R. C. (1971). The assessment and analysis of handedness: the Edinburgh inventory. Neurophychologia 9, 97-113. doi: 10.1016/0028-3932(71)90067-4

Palmer, C. J., Paton, B., Kirkovski, M., Enticott, P. G., and Hohwy, J. (2015a). Context sensitivity in action decreases along the autism spectrum: a predictive processing perspective. Proc. Biol. Sci. 282:20141557. doi: 10.1098/rspb. 2014.1557

Palmer, C. J., Paton, B., Hohwy, J., and Enticott, P. G. (2013). Movement under uncertainty: the effects of the rubber-hand illusion vary along the nonclinical autism spectrum. Neuropsychologia 51, 1942-1951. doi: 10.1016/j.neuropsychologia.2013.06.020

Palmer, C. J., Paton, B., Enticott, P. G., and Hohwy, J. (2015b). 'Subtypes' in the presentation of autistic traits in the general adult population. J. Autism Dev. Disord. 45, 1291-1301. doi: 10.1007/s10803-014-2289-1

Paton, B., Hohwy, J., and Enticott, P. G. (2012). The rubber hand illusion reveals proprioceptive and sensorimotor differences in autism spectrum disorders. J. Autism Dev. Disord. 42, 1870-1883. doi: 10.1007/s10803-011-1430-7

Pineles, S. L., and Mineka, S. (2005). Attentional biases to internal and external sources of potential threat in social anxiety. J. Abnorm. Psychol. 114, 314-318. doi: 10.1037/0021-843X.114.2.314

Schauder, K. B., Mash, L. E., Bryant, L. K., and Cascio, C. J. (2015). Interoceptive ability and body awareness in autism spectrum disorder. J. Exp. Child Psychol. 131, 193-200. doi: 10.1016/j.jecp.2014.11.002

Shimada, S., Fukuda, K., and Hiraki, K. (2009). Rubber hand illusion under delayed visual feedback. PLoS ONE 4:e6185. doi: 10.1371/journal.pone.0006185

Shimada, S., Suzuki, T., Yoda, N., and Hayashi, T. (2014). Relationship between sensitivity to visuotactile temporal discrepancy and the rubber hand illusion. Neurosci. Res. 85, 33-38. doi: 10.1016/j.neures.2014. 04.009

Suzuki, K., Garfinkel, S. N., Critchley, H. D., and Seth, A. K. (2013). Multisensory integration across exteroceptive and interoceptive domains modulates selfexperience in the rubber-hand illusion. Neuropsychologia 51, 2909-2917. doi: 10.1016/j.neuropsychologia.2013.08.014

Tsakiris, M., and Haggard, P. (2005). The rubber hand illusion revisited: visuotactile integration and self-attribution. J. Exp. Psychol. Hum. Percept. Perform. 31, 80-91. doi: 10.1037/0096-1523.31.1.80

Tsakiris, M., Tajadura-Jimenéz, A., and Costantini, M. (2011). Just a heartbeat away from one's body: interoceptive sensitivity predicts malleability of bodyrepresentations. Proc. Biol. Sci. 278, 2470-2476. doi: 10.1098/rspb.2010.2547

Wakabayashi, A., Tojo, Y., Baron-Cohen, S., and Wheelwright, S. (2004). The Autism-Spectrum Quotient(AQ)Japanese version:Evidence from highfunctioning clinical group and normaladults. Jpn. J. Psychol. 75, 78-84. doi: 10.4992/jjpsy.75.78

Conflict of Interest Statement: The authors declare that the research was conducted in the absence of any commercial or financial relationships that could be construed as a potential conflict of interest.

Copyright (C) 2016 Ide and Wada. This is an open-access article distributed under the terms of the Creative Commons Attribution License (CC BY). The use, distribution or reproduction in other forums is permitted, provided the original author(s) or licensor are credited and that the original publication in this journal is cited, in accordance with accepted academic practice. No use, distribution or reproduction is permitted which does not comply with these terms. 